\title{
Permissive Dynamic Information Flow Analysis
}

\author{
Thomas H. Austin \\ University of California, Santa Cruz \\ 1156 High Street \\ Santa Cruz, CA 95064, United States \\ taustin@ucsc.edu
}

\author{
Cormac Flanagan \\ University of California, Santa Cruz \\ 1156 High Street MS: SOE3 \\ Santa Cruz, CA 95064, United States \\ cormac@ucsc.edu
}

\begin{abstract}
A key challenge in dynamic information flow analysis is handling implicit flows, where code conditional on a private variable updates a public variable $\mathrm{x}$. The naive approach of upgrading $\mathrm{x}$ to private results in $\mathrm{x}$ being partially leaked, where its value contains private data but its label might remain public on an alternative execution (where the conditional update was not performed). Prior work proposed the no-sensitive-upgrade check, which handles implicit flows by prohibiting partially leaked data, but attempts to update a public variable from a private context causes execution to get stuck.

To overcome this limitation, we develop a sound yet flexible permissive-upgrade strategy. To prevent information leaks, partially leaked data is permitted but carefully tracked to ensure that it is never totally leaked. This permissiveupgrade strategy is more flexible than the prior approaches such as the no-sensitive-upgrade check.

Under the permissive-upgrade strategy, partially leaked data must be marked as private before being used in a conditional test, thereby ensuring that it is private for both the current execution as well as alternate execution paths. This paper also presents a dynamic analysis technique for inferring these privatization operations and inserting them into the program source code. The combination of these techniques allows more programs to run to completion, while still guaranteeing termination-insensitive non-interference in a purely dynamic manner.
\end{abstract}

\section{Categories and Subject Descriptors}

D.3.3 [Programming Languages]: Language Constructs and Features; D.4.6 [Operating Systems]: Security and Protection-Information flow controls

\section{General Terms}

Languages, Security

Permission to make digital or hard copies of all or part of this work for personal or classroom use is granted without fee provided that copies are not made or distributed for profit or commercial advantage and that copies bear this notice and the full citation on the first page. To copy otherwise, to republish, to post on servers or to redistribute to lists, requires prior specific permission and/or a fee.

PLAS ' 10 Toronto, Canada

Copyright 2010 ACM 978-1-60558-827-8 ...\$10.00.

\section{Keywords}

Information flow control, dynamic analysis

\section{INTRODUCTION}

JavaScript has become the dominant language for clientside web development. Once relegated to form validation and similar small tasks, JavaScript today has become a major component of the Web 2.0 architecture; applications such as Google Maps and Gmail rely on it heavily to give online applications the interactive features previously limited to the realm of desktop applications. Browser vendors have spent a good deal of effort on their JavaScript implementations, so that recent versions have become tremendously fast [17].

But as JavaScript's role has grown, its security vulnerabilities have become more significant. Most prominently, cross-site scripting (XSS) has become one of the most pervasive computer security vulnerabilities. Mashups [26], where code is combined from multiple sites, are particularly problematic, and yet they are very popular. In response, a wide array of security mechanisms have been put in place. The same origin policy [29] is one of the oldest, beginning with early versions of Netscape. It prevents scripts from accessing documents from other domains, but it does very little to control the interaction of scripts loaded in the same page. To give developers greater freedom, Mozilla developed a system for signed scripts [28] and Internet Explorer created Security Zones [27]. Unfortunately, the permissions granted by these two systems have little overlap, making developing secure applications that function correctly across all browsers extremely difficult. Other strategies have involved limiting JavaScript to only a subset of language features; this is the approach taken by Facebook with FBJS [15] and Google with Caja [18]. This list covers only a portion of the total security mechanisms focused on JavaScript and the browser.

The error-prone nature of software systems suggests that critical security policies are best enforced by small trusted modules, rather than being an emergent property of complex and buggy application code. Just as memory-safe languages provide a resilient defense against buffer-overrun vulnerabilities, violations of privacy or data integrity expectations need a similar systemic solution. While these concerns apply to a wide variety of programs, they are particularly relevant in a browser setting where code fragments from multiple untrusted or semi-trusted servers execute within the same process.

Information flow analysis is a compelling option for solving these issues. It gives a stronger guarantee that confidentiality and integrity are protected, while being arguably less 


\begin{tabular}{|c|c|c|c|c|}
\hline \multirow[b]{2}{*}{ Function $\mathrm{f}(\mathrm{x})$} & $\mathrm{x}=\mathrm{fals} \mathrm{e}^{H}$ & \multicolumn{3}{|c|}{$\mathrm{x}=\operatorname{true}^{H}$} \\
\hline & All strategies & Naive & No-Sensitive-Upgrade & Permissive-Upgrade \\
\hline $\begin{array}{l}y=\text { true; } \\
z=\text { true; } \\
\text { if }(x) \\
\quad y=\text { false; } \\
\text { if }(y) \\
\quad z=\text { false; } \\
\text { return } z ;\end{array}$ & $\begin{array}{c}\mathrm{y}=\text { true }^{\mathrm{L}} \\
\mathrm{z}=\text { true }^{\mathrm{L}} \\
\text { branch not taken } \\
\text { y remains true } \\
\text { branch taken, } p c=L \\
\mathrm{z} \text { updated to } \mathrm{false}^{L} \\
\text { returns false }\end{array}$ & $\begin{array}{c}\mathrm{y}=\text { true }^{\mathrm{L}} \\
\mathbf{z}=\text { true }^{\mathrm{L}} \\
\text { branch taken, } p c=H \\
\mathrm{y} \text { updated to false }{ }^{H} \\
\text { branch not taken } \\
\mathrm{z} \text { remains true } \\
\text { returns true }\end{array}$ & $\begin{array}{c}\mathrm{y}=\text { true }^{\mathrm{L}} \\
\mathbf{z}=\text { true }^{\mathrm{L}} \\
\text { branch taken, } p c=H \\
\text { stuck }\end{array}$ & $\begin{array}{c}\mathrm{y}=\text { true }^{\mathrm{L}} \\
\mathrm{z}=\text { true }^{\mathrm{L}} \\
\text { branch taken, } p c=H \\
\text { y updated to false }{ }^{P} \\
\text { stuck, infer upgrade }\end{array}$ \\
\hline Return Value: & false $e^{L}$ & true $^{L}$ & & \\
\hline
\end{tabular}

restrictive than some measures currently being used. Much prior work has focused on providing information flow security guarantees via type-based static analyses [41, 21, 8, 42, 31]. In general, static analyses are often preferred for their advantages in performance and because of their ability to reason about all paths of execution. Unfortunately, typebased static analyses are not applicable to browser-based applications written in JavaScript, which is a dynamically typed language. Therefore our work focuses on enforcing information flow policies dynamically rather than statically.

Previous work has addressed some of the performance concerns of dynamic analysis [4], but verifying information flow properties via a purely dynamic analysis is rather tricky. The central correctness property that we wish to enforce is termination-insensitive non-interference, which says that changing the private inputs to an application should not influence any of the public outputs. ${ }^{1}$ Verifying this property dynamically requires simultaneously reasoning about the current actual execution of the program, as well as possible alternate executions of the program on the same public inputs but different private inputs.

Dynamic analysis can reason precisely about the actual execution, but simultaneously reasoning about possible alternate executions is rather difficult, particularly when the alternate execution could execute different code and update different memory locations than the actual execution. A particular challenge is handling implicit flows, when code whose execution is conditional on private information updates a public variable.

The code fragment in Figure 1 captures the essence of this difficulty in a simple example. This code defines a function $f$ that takes a private boolean argument $\mathrm{x}$, initializes two public variables y and $\mathbf{z}$ to true, and then conditionally updates both of these variables before returning $z$. Thus, information flows from the private argument variable $\mathrm{x}$ into $\mathrm{y}$ and then into $\mathbf{z}$, and the challenge is to track this information flow dynamically so that $\mathbf{z}$ is also labeled as private. The security label $H$ denotes private or high confidentiality data, and conversely $L$ denotes public or low confidentiality data. Tracking the information flow due to a conditional assignment that does not happen is particularly difficult, as we discuss below.

\footnotetext{
${ }^{1}$ As in other approaches, the termination channel may leak one bit of data, or somewhat more in the presence of intermediary outputs [1].
}

Naive. An intuitive (but ineffective) strategy for handling the first conditional assignment to y is to upgrade the label on y to $H$, since that assignment is conditional on the private variable $\mathrm{x}$. In the case where $\mathrm{x}$ is $\operatorname{true}^{H}$ then $\mathrm{y}$ becomes $\mathrm{false}^{H}$, and is appropriately labeled private; however, if $\mathrm{x}$ is false ${ }^{H}$ then y remains true ${ }^{L}$ and is still labeled public. Thus, we say that the variable $\mathrm{y}$ is partially leaked, since $\mathrm{y}$ now contains private information but $\mathrm{y}$ is labeled private on only one of these two executions.

Continuing the example, we now perform a second conditional assignment to $\mathbf{z}$, which is initially true ${ }^{L}$. The result of these two conditionals is that $\mathbf{z}$ is labeled public, but contains the value of the private input $x$. That is, if $\mathrm{x}$ is true ${ }^{H}$ then $\mathrm{y}$ becomes $\mathrm{false}^{H}$ and $\mathrm{z}$ remains true ${ }^{L}$; conversely, if $\mathrm{x}$ is $\mathrm{false}^{H}$ then $\mathrm{y}$ remains true ${ }^{L}$ and so $\mathrm{z}$ becomes false ${ }^{L}$. Thus, the naive approach to handling implicit flows permits both partially leaked data (in y) and totally leaked data (in $\mathbf{z}$ ), and fails to provide terminationinsensitive non-interference.

No-Sensitive-Upgrade. The above intuitive approach of simply upgrading the security label of the conditionally assigned variable is inadequate. A proposed solution uses the no-sensitive-upgrade check [42, 4], whereby execution will fail-stop or get stuck whenever data would be partially leaked. Under this strategy, the assignment to the public variable $\mathrm{y}$ from code conditional on a private variable $\mathrm{x}$ would get stuck.

Although this strategy satisfies termination-insensitive noninterference, it also rejects valid programs that have no information leak. To illustrate this limitation, consider the following code snippet where the input $\mathrm{x}$ is private:

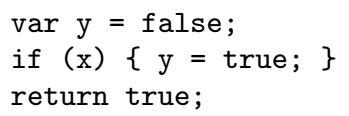

Although no information leak occurs, this program gets stuck under the no-sensitive-upgrade approach (and would also be rejected by many static analyses).

Permissive-Upgrade. The goal of this paper is to allow more applications to run to completion than under the no-sensitive-upgrade check, while still providing information flow security guarantees.

Our proposed permissive-upgrade strategy tolerates and carefully tracks partially leaked data, while still providing termination-insensitive non-interference. The central idea 
Figure 2: Implicit flow function with privatization operation

\begin{tabular}{|c|c|c|}
\hline \multirow[b]{2}{*}{ Function $\mathrm{f}(\mathrm{x})$} & \multicolumn{2}{|c|}{ Permissive-Upgrade } \\
\hline & $\mathrm{x}=\mathrm{false} \mathrm{f}^{H}$ & $\mathrm{x}=$ true $^{H}$ \\
\hline $\begin{array}{l}y=\text { true; } \\
z=\text { true; } \\
\text { if }(x) \\
\quad y=f a l s e ; \\
\text { if }(<H>y) \\
\quad z=f a l s e ; \\
\text { return } z ;\end{array}$ & $\begin{array}{c}\mathrm{y}=\text { true }^{L} \\
\mathbf{z}=\text { true }^{L} \\
\text { branch not taken } \\
\text { y remains true } \\
\text { branch taken, } p c=H \\
\text { z updated to fals } e^{P} \\
\text { returns false }{ }^{P}\end{array}$ & $\begin{array}{c}\mathrm{y}=\text { true }^{L} \\
\mathbf{z}=\text { true }^{L} \\
\text { branch taken, } p c=H \\
\text { y updated to false }{ }^{P} \\
\text { branch not taken } \\
\mathbf{z} \text { remains true }{ }^{L} \\
\text { returns true }\end{array}$ \\
\hline Return Value: & false ${ }^{P}$ & true $^{L}$ \\
\hline
\end{tabular}

is to introduce an additional label $P$ to identify and track partially leaked data:

The security label $P$ identifies partially leaked data that contains private information but which may be labeled as public in some alternative executions.

Thus, at the conditional assignment to $\mathrm{y}$ in Figure 1, if $\mathrm{x}$ is false ${ }^{H}$ then y remains true ${ }^{L}$, as the assignment is not performed. If $\mathrm{x}$ is true $^{H}$, however, then $\mathrm{y}$ is updated to false ${ }^{P}$, where the label $P$ reflects that in other executions y may remain labeled public.

Such partially leaked data must be handled quite delicately. In particular, if y is ever used in a conditional branch, as in the second conditional of Figure 1, then the permissiveupgrade strategy still gets stuck in order to avoid converting a partial information leak into a total information leak.

To avoid getting stuck in this situation, the conditional test expression y can be labeled as private before the conditional test, as shown in Figure 2. This privatization operation

\section{$\langle H\rangle \mathrm{y}$}

converts both public $(L)$ and partially leaked $(P)$ data to private $(H)$. Critically, converting partially leaked data to private is sound since, as a consequence of the labeling operation, the resulting data is made private on all executions, including alternative executions where y was originally labeled public. Thus, we can avoid stuck executions simply by inserting privatization operations at all sensitive uses of partially leaked data. Sensitive uses include conditional branches, as described above, but also other operations such as indirect jumps, virtual method calls, etc. Once all the necessary privatization operations are in place, program execution will never fail-stop (although it may diverge). Any results returned will be labeled in a way that accounts for any influence from private data, including via implicit flows.

Privatization Inference. Finding all of these sensitive use points manually, however, can be an onerous task. This overhead is problematic since convincing developers to adopt different security tools is always something of a challenge. Especially when extra work is required, resistance to adoption can be fierce.

Fortunately, we can extend the permissive-upgrade semantics to minimize the burden placed on developers. Whenever a program would get stuck based on a sensitive use of partially leaked data, the runtime engine can infer the needed privatization operations. Over time, these privatization operations will improve the precision of the analysis, rejecting fewer program executions.

We present an extension of our permissive-upgrade evaluation semantics that also infers these privatization operations. In situations where our original semantics would get stuck because of a sensitive use of partially leaked data, the extended semantics automatically inserts the appropriate privatization operation instead, and so continues execution. Thus, the conditional test "if (y)" is automatically converted to "if $(\langle H\rangle \mathrm{y})$ ".

In practice, we envision that these techniques could be applied as follows: A JavaScript web application is initially released in an instrumented form that uses the extended semantics to infer the needed privatization operations. This semantics never gets stuck but does not (yet) provide information flow guarantees. After a certain period of testing, most privatization operations will be determined, and the appropriately modified application could be re-released under the permissive-upgrade semantics with strong informationflow guarantees. Subsequently, some executions may still get stuck, but these are likely to be few, and can immediately be used to update the privatization operations for the application, preventing subsequent executions from getting stuck at the same sensitive operation. In this manner, the difficulty of inferring the correct privatization operations can be amortized over a large collection of users.

We hope that these inference techniques may help migrate existing Javascript web applications into a more secure world, where information flow policies are tracked and enforced by the language runtime itself. This deployment strategy requires information-flow support in the browser's JavaScript implementation, and we are exploring how to incorporate such extensions in the Firefox browser [14].

\section{A CORE LANGUAGE FOR INFORMATION FLOW}

We formalize our permissive-upgrade strategy in terms of $\lambda^{\text {info }}$, an imperative extension of the lambda calculus described in Figure 3. The lambda calculus has a rich tradition as a foundational test-bed for research in programming languages and type theory, and we believe that it is an equally effective platform for investigating information flow security.

Terms include variables $(x)$, constants $(c)$, functions $(\lambda x . e)$, and function application $\left(e_{1} e_{2}\right)$. Constants include integers as well as primitive operations such as "+". Since many of the challenges in information flow analysis come from imperative updates, our language supports mutable reference cells, including terms for allocating (ref $e$ ), dereferencing $(! e)$, and updating $\left(e_{1}:=e_{2}\right)$ a reference cell. Finally, there is a term for labeling data as private $(\langle H\rangle e)$.

$\lambda^{\text {info }}$ is much simpler than JavaScript, allowing us to reason more easily about some of the challenges involved in correctly handling implicit flows. Although exceptions add important additional complexities to implicit flows [2, 24], we leave them for future work.

Many additional constructs can be built from this core language; the second part of Figure 3 sketches some standard encodings for booleans, conditionals, let-expressions, and sequential composition.

As an illustrative example of $\lambda^{\text {info }}$, Figure 4 translates the 
Figure 3: The source language $\lambda^{\text {info }}$

Syntax:

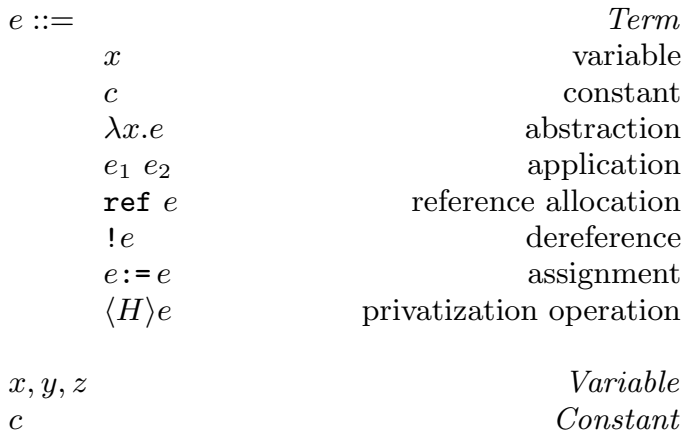

Standard encodings:

$$
\begin{array}{rll}
\text { true } & \stackrel{\text { def }}{=} \lambda x \cdot \lambda y \cdot x \\
\text { false } & \stackrel{\text { def }}{=} \lambda x \cdot \lambda y . y \\
\text { if } e_{1} \text { then } e_{2} \text { else } e_{3} & \stackrel{\text { def }}{=}\left(e_{1}\left(\lambda d . e_{2}\right)\left(\lambda d . e_{3}\right)\right)(\lambda x . x) \\
\text { if } e_{1} \text { then } e_{2} & \stackrel{\text { def }}{=} \text { if } e_{1} \text { then } e_{2} \text { else } 0 \\
\text { let } x=e_{1} \text { in } e_{2} & \stackrel{\text { def }}{=}\left(\lambda x \cdot e_{2}\right) e_{1} \\
e_{1} ; e_{2} & \stackrel{\text { def }}{=} \text { let } x=e_{1} \text { in } e_{2}, \quad x \notin F V\left(e_{2}\right)
\end{array}
$$

Figure 4: The implicit flow function $f$ in $\lambda^{\text {info }}$

$\lambda \mathrm{x}$

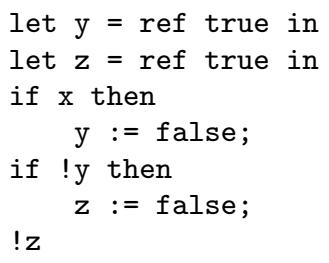

implicit flow function $\mathrm{f}(\mathrm{x})$ shown in Figure 1 from JavaScript into $\lambda^{\text {info }}$. The translated function proceeds in an analogous manner to the original function, except that JavaScript mutable variables are now represented as reference cells. The $\lambda^{\text {info }}$ version creates two public reference cells $\mathrm{y}$ and $\mathrm{z}$ and conditionally updates both of them. It then returns the value of the reference cell $\mathbf{z}$ via the dereference operation !z.

\section{THREE EVALUATION STRATEGIES}

We next formalize the permissive-upgrade evaluation strategy for the idealized language $\lambda^{\text {info }}$. For completeness, we also formalize the two other evaluation strategies (naive and no-sensitive-upgrade) discussed in the introduction. Figure 5 presents the core semantics that is common to all evaluation strategies.

The semantics includes both public $(L)$ and private $(H)$ labels, as well as the partially leaked label $(P)$, which is used exclusively by the permissive-upgrade semantics. In a more general setting with multiple principals, each security label would have the type

$$
\text { Principal } \rightarrow\{L, H, P\} .
$$

Our approach extends to this more general setting, but for clarity of exposition we present our ideas in a simpler setting with just a single principal and a three element label lattice. Labels are ordered by

$$
L \sqsubseteq H \sqsubseteq P
$$

reflecting the constraints on how correspondingly labeled data is used, noting that partially leaked data must be handled in a more restrictive manner than private data. We use $\sqcup$ to denote the corresponding join operation on labels. Critically, because $P$ is more restrictive than $H, H \sqcup P=P$.

In the evaluation semantics, each reference cell is allocated at an address $a$. A store $\sigma$ maps addresses to values. A raw value $r$ is either a constant $(c)$, an address $(a)$, or a closure $(\lambda x . e, \theta)$, which is a pair of a $\lambda$-expression and a substitution $\theta$ that maps variables to values. A value $v$ has the form $r^{k}$, which combines both an information flow label $k \in\{L, H, P\}$ and a raw value $r$. We use $\emptyset$ to denote both the empty store and the empty substitution.

Figure 5 defines the semantics of $\lambda^{\text {info }}$ via the big-step evaluation relation:

$$
\sigma, \theta, e \Downarrow_{p c} \sigma^{\prime}, v
$$

This relation evaluates an expression $e$ in the context of a store $\sigma$, a substitution $\theta$, and the current label $p c$ of the program counter, and returns the resulting value $v$ and the (possibly modified) store $\sigma^{\prime}$. The program counter label $p c \in\{L, H\}$ reflects whether the execution of the current code is conditional on private data.

The rules defining this evaluation relation are straightforward, with some notable subtleties on how labels are handled. In particular, we adopt the invariant that the label on the resulting value $v$ is at least as secret as the program counter $(p c \sqsubseteq$ label $(v))$. Thus, for example, the [CONST] rule evaluates a const $c$ to the labeled value $c^{p c}$. The [Fun] rule evaluates a function $(\lambda x . e)$ to a closure $(\lambda x . e, \theta)^{p c}$ that captures the current substitution and that includes the program counter label. The $[\mathrm{VAR}]$ rule for a variable reference $x$ extracts the corresponding value $\theta(x)$ from the environment and strengthens its label to be at least $p c$, using the following overloading of the join operator:

$$
\left(r^{l}\right) \sqcup k \stackrel{\text { def }}{=} \quad r^{(l \sqcup k)}
$$

The [APP] rule applies a closure to an argument; to avoid information leaks, this rule gets stuck if the closure is partially leaked. The [PRIM] rule applies function primitives. The [REF] and [DEREF] rules create and dereference a reference cell, respectively.

The [LABEL] rule for $\langle H\rangle e$ explicitly tags the result of evaluating $e$ as private, ignoring the original label $k$. This rule can be used either to upgrade public data or downgrade partially leaked data. Note that the latter case is safe, since the data will be made private on the current execution as well as any alternate execution.

From these rules, we can derive corresponding evaluation rules for the encoded constructs, which are also shown in Figure 5. Critically, the [THEN] and [ELSE] rules get stuck if the conditional is partially leaked. 
Figure 5: Core semantics for $\lambda^{\text {info }}$

Runtime Syntax:

$\begin{array}{rllll}a & \in & \text { Address } & & \\ \sigma & \in & \text { Store } & = & \text { Address } \rightarrow p \text { Value } \\ \theta & \in & \text { Subst } & = & \text { Var } \rightarrow p \text { Value } \\ r & \in & \text { RawValue } & ::= & c|a|(\lambda x . e, \theta) \\ v & \in & \text { Value } & ::= & r^{k} \\ k, l, p c & \in & \text { Label } & ::= & L|H| P\end{array}$

Evaluation Rules: $\quad \sigma, \theta, e \Downarrow_{p c} \sigma^{\prime}, v$

[CONST]

[FUN]

$$
\overline{\sigma, \theta, c \Downarrow_{p c} \sigma, c^{p c}}
$$$$
\overline{\sigma, \theta,(\lambda x . e) \Downarrow_{p c} \sigma,(\lambda x . e, \theta)^{p c}}
$$

[VAR]

$$
\overline{\sigma, \theta, x \Downarrow_{p c} \sigma,(\theta(x) \sqcup p c)}
$$

[LABEL]

$$
\frac{\sigma, \theta, e \Downarrow_{p c} \sigma^{\prime}, r^{k}}{\sigma, \theta,\langle H\rangle e \Downarrow_{p c} \sigma^{\prime}, r^{H}}
$$

[APP]

$$
\begin{gathered}
\sigma, \theta, e_{1} \Downarrow_{p c} \sigma_{1},\left(\lambda x . e, \theta^{\prime}\right)^{k} \\
k \neq P \\
\sigma_{1}, \theta, e_{2} \Downarrow_{p c} \sigma_{2}, v_{2} \\
\sigma_{2}, \theta^{\prime}\left[x:=v_{2}\right], e \Downarrow_{k} \sigma^{\prime}, v \\
\hline \sigma, \theta,\left(e_{1} e_{2}\right) \Downarrow_{p c} \sigma^{\prime}, v
\end{gathered}
$$

[PRIM]

$$
\begin{gathered}
\sigma, \theta, e_{1} \Downarrow_{p c} \sigma_{1}, c^{k} \\
\sigma_{1}, \theta, e_{2} \Downarrow_{p c} \sigma_{2}, d^{l} \\
r=\llbracket c \rrbracket(d) \\
\frac{r, \theta,\left(e_{1} e_{2}\right) \Downarrow_{p c} \sigma_{2}, r^{k \sqcup l}}{\sigma,}
\end{gathered}
$$

[REF]

$$
\begin{gathered}
\sigma, \theta, e \Downarrow_{p c} \sigma^{\prime}, v \\
\frac{a \notin \operatorname{dom}\left(\sigma^{\prime}\right)}{\sigma, \theta,(\operatorname{ref} e) \Downarrow_{p c} \sigma^{\prime}[a:=v], a^{p c}}
\end{gathered}
$$

[DEREF]

$$
\frac{\sigma, \theta, e \Downarrow_{p c} \sigma^{\prime}, a^{k}}{\sigma, \theta, ! e \Downarrow_{p c} \sigma^{\prime},\left(\sigma^{\prime}(a) \sqcup k\right)}
$$

\section{Derived Evaluation Rules:}

$$
\begin{aligned}
& \text { [THEN] } \\
& \sigma, \theta, e_{1} \Downarrow_{p c} \sigma_{1},(\text { true }, \theta)^{k} \\
& k \neq P \\
& \sigma_{1}, \theta, e_{2} \Downarrow_{k} \sigma^{\prime}, v \\
& \overline{\sigma, \theta,\left(\text { if } e_{1} \text { then } e_{2} \text { else } e_{3}\right) \Downarrow_{p c} \sigma^{\prime}, v}
\end{aligned}
$$

[LET]

$$
\begin{gathered}
\sigma, \theta, e_{1} \Downarrow_{p c} \sigma_{1}, v_{1} \\
\sigma_{1}, \theta\left[x:=v_{1}\right], e_{2} \Downarrow_{p c} \sigma^{\prime}, v \\
\sigma, \theta,\left(\text { let } x=e_{1} \text { in } e_{2}\right) \Downarrow_{p c} \sigma^{\prime}, v
\end{gathered}
$$

[ELSE]

$$
\sigma, \theta, e_{1} \Downarrow_{p c} \sigma_{1},(\text { false }, \theta)^{k}
$$$$
k \neq P
$$

$\sigma_{1}, \theta, e_{3} \Downarrow_{k} \sigma^{\prime}, v$

$\overline{\sigma, \theta,\left(\text { if } e_{1} \text { then } e_{2} \text { else } e_{3}\right) \Downarrow_{p c} \sigma^{\prime}, v}$

[SEQ]

$$
\begin{gathered}
\sigma, \theta, e_{1} \Downarrow_{p c} \sigma_{1}, v_{1} \\
\sigma_{1}, \theta, e_{2} \Downarrow_{p c} \sigma^{\prime}, v \\
\hline \sigma, \theta,\left(e_{1} ; e_{2}\right) \Downarrow_{p c} \sigma^{\prime}, v
\end{gathered}
$$


Figure 6: A secure function

\begin{tabular}{|c|c|c|c|}
\hline \multirow[t]{2}{*}{ Function $\mathrm{g}(\mathrm{x})$} & $\mathrm{x}=\mathrm{false} \mathrm{s}^{H}$ & \multicolumn{2}{|c|}{$\mathrm{x}=\operatorname{true}^{H}$} \\
\hline & Both & $N S U$ & Perm. $U$. \\
\hline $\begin{array}{l}\text { let } y=\text { ref true in } \\
\text { if } x \text { then } y:=f a l s e ; \\
y:=\text { true; } \\
y\end{array}$ & $\begin{array}{l}\text { true }^{L} \\
\text { true }^{L} \\
\text { true }^{L}\end{array}$ & $\begin{array}{l}\text { true }^{L} \\
\text { stuck }\end{array}$ & $\begin{array}{l}\text { true }^{L} \\
\text { false } \\
\text { true }^{L}\end{array}$ \\
\hline Return Value: & true $^{L}$ & & true $^{L}$ \\
\hline
\end{tabular}

Assignment statements are notably missing from Figure 5 since they introduce difficult problems with implicit flows. Below, we formalize the three strategies for tracking implicit flows as three different rules for evaluating assignment statements.

We also illustrate these strategies on the example function $f(x)$ shown in Figure 4. In the situation where the argument $\mathrm{x}$ is $\mathrm{false} \mathrm{s}^{H}$, all three evaluation strategies return false ${ }^{L}$. The following subsections describe how different strategies handle the tricky case where $\mathrm{x}$ is true $^{H}$ and where $\mathrm{f}$ must update the public reference cell y.

\subsection{The Naive Approach}

The intuitive approach for assignment is to promote the label on the reference cell to at least the label $k$ on the address $a^{k}$. (Note that a global evaluation invariant ensures that $p c \sqsubseteq k$.)

$$
\begin{gathered}
\sigma, \theta, e_{1} \Downarrow_{p c} \sigma_{1}, a^{k} \\
\frac{\sigma_{1}, \theta, e_{2} \Downarrow_{p c} \sigma_{2}, v}{\sigma, \theta,\left(e_{1}:=e_{2}\right) \Downarrow_{p c} \sigma_{2}[a:=(v \sqcup k)], v} \quad \text { [ASSIGN-NAIVE] }
\end{gathered}
$$

For the function call $f\left(\right.$ true $\left.^{H}\right)$, this strategy updates y to false ${ }^{H}$ but leaves $\mathbf{z}$ as true ${ }^{L}$. Thus, by comparing the return value for the All strategies and Naive column of Figure 1 , we see that the result of $f(x)$ is a publicly labeled copy of its private argument, and so this naive approach leaks information.

\subsection{The No-Sensitive-Upgrade Approach}

The no-sensitive-upgrade (NSU) approach avoids information leaks by getting stuck if a public reference cell is updated when the $p c$ is private, or when the label on the target address is private. (In an implementation such stuck states might cause an exception to be thrown to the top level.)

The following rule requires that the label $k$ on the target address $a^{k}$ is at most the label on the reference cell contents. This rule assumes all data is labeled public or private, but never partially leaked.

$$
\begin{gathered}
\sigma, \theta, e_{1} \Downarrow_{p c} \sigma_{1}, a^{k} \\
\sigma_{1}, \theta, e_{2} \Downarrow_{p c} \sigma_{2}, v \\
k \sqsubseteq \operatorname{label}\left(\sigma_{2}(a)\right) \\
\frac{}{\sigma, \theta,\left(e_{1}:=e_{2}\right) \Downarrow_{p c} \sigma_{2}[a:=(v \sqcup k)], v}
\end{gathered}
$$

For our example function, the call $f\left(\operatorname{true}^{H}\right)$ would get stuck on the update to the public variable y within a private branch of execution, as illustrated by the NSU column of Figure 1, preventing the information leak.
Unfortunately, the NSU strategy may also get stuck on code that does not leak information, as shown in Figure 6. Although there is no information leak, evaluation of $\mathrm{g}\left(\right.$ true $\left.^{H}\right)$ gets stuck when the private parameter $\mathrm{x}$ is partially leaked. Thus, the NSU strategy satisfies termination-insensitive noninterference, but is unnecessarily restrictive.

\subsection{The Permissive-Upgrade Approach}

The permissive-upgrade semantics introduces an additional label $(P)$ in order to tolerate and track partially leaked data. This strategy allows us to defer the point of failure and reduce the number of false positives.

The rule [ASSIGN-PERMissive] below considers an assignment to an address $a^{k}$ that currently holds a value labeled $l$. The rule requires that the address is not partially leaked $(k \neq P)$.

$$
\begin{gathered}
\text { [ASSIGN-PERMISSIVE] } \\
\sigma, \theta, e_{1} \Downarrow_{p c} \sigma_{1}, a^{k} \\
\sigma_{1}, \theta, e_{2} \Downarrow_{p c} \sigma_{2}, v \\
l=\operatorname{label}\left(\sigma_{2}(a)\right) \\
k \neq P \\
m=\operatorname{lift}(k, l) \\
\frac{m, \theta,\left(e_{1}:=e_{2}\right) \Downarrow_{p c} \sigma_{2}[a:=(v \sqcup m)], v}{}
\end{gathered}
$$

The rule uses the following function $\operatorname{lift}(k, l)$ to infer the new label $m$ for the reference cell.

\begin{tabular}{|c|c||c|}
\hline$k$ & $l$ & lift $(k, l)$ \\
\hline$L$ & any & $L$ \\
$H$ & $L$ & $P$ \\
$H$ & $H$ & $H$ \\
$H$ & $P$ & $P$ \\
\hline
\end{tabular}

We consider each possible combination of labels $k$ and $l$ :

- If the target address is public $(k=L)$, then execution is not in a private context (due to the evaluation invariant that $p c \sqsubseteq k$ ). In this situation there are no difficulties with implicit flows, so $m=L$.

- Conversely, if the target address or execution context is private $(k=H)$, then an attempt to update a public reference cell $(l=L)$ results in the new contents being labeled as partially leaked $(m=P)$.

- Updating a private cell from a private context is fine, and results in a private cell.

- Finally, updating a partially leaked cell from a private context leaves the cell as partially leaked.

For the function call $\mathrm{f}\left(\mathrm{true}^{H}\right)$ from Figure 1, the permissiveupgrade strategy handles the first conditional assignment by marking y as partially leaked, but gets stuck on the second conditional test, to avoid information leaks.

We can remedy this situation by introducing the label $\langle H\rangle$ :

$$
\text { if }(\langle H\rangle \text { !y) then } \mathrm{z}:=\text { false; }
$$

This privatization operation ensures the test expression is private on both executions, rather than partially leaked on 
one execution and public on the other. The modified function $f$ now runs to completion on all boolean inputs. Section 5 discusses how to infer these privatization operations automatically.

Figure 6 demonstrates that, under the permissive-upgrade strategy, the function $g$ runs to completion on all boolean inputs (unlike under NSU). More generally, the following theorem shows that any execution that does not get stuck under NSU evaluation (denoted $\Downarrow_{p c}^{\text {nu }}$ ) will also not get stuck under permissive-upgrade evaluation (denoted $\Downarrow_{p c}$ ). Thus, the permissive-upgrade strategy is strictly superior to NSU. For the proof of this theorem, we refer the interested reader to a related technical report [5].

TheOREM 1. Suppose $\sigma, \theta$, and pc do not contain the partially leaked label $P$ and $\sigma, \theta, e \Downarrow_{p c}^{\text {nu }} \sigma^{\prime}, v$. Then $\sigma, \theta, e \Downarrow_{p c}$ $\sigma^{\prime}, v$, and $\sigma^{\prime}$ and $v$ do not contain $P$.

Partially leaked data must be handled carefully, since on an alternative execution this data might be labeled as public. In particular, function calls, conditionals, and assignments are considered sensitive operations; these operations get stuck (via the antecedent $k \neq P$ ) if applied to partially leaked data (as otherwise our information flow analysis could not track how alternative executions may propagate partially leaked information). These stuck sensitive operations are critical for avoiding information leaks, and they distinguish the permissive-upgrade approach from the unsound naive approach.

To motivate why assignment statements are sensitive operations, consider the function $\mathrm{h}(\mathrm{x})$ shown in Figure 7 . This function allocates two reference cells $y$ and $z$, initializes $w$ as a pointer to $\mathrm{y}$, and then, depending on the private argument $\mathrm{x}$, conditionally updates $\mathrm{w}$ to point to $\mathrm{z}$. At this stage, $\mathrm{w}$ is partially leaked, since whether it points to $\mathrm{y}$ or $\mathbf{z}$ depends on the input argument $\mathrm{x}$. Updating the reference cell pointed to by w would result in totally leaked data, and must be precluded by the evaluation getting stuck at the indirect assignment

$$
\text { (!w) := false }
$$

as shown in the third column of Figure 7.

The right hand side of Figure 7 illustrates how privatization operations overcome this limitation. The new function $\mathrm{h}$ _priv is identical to $\mathrm{h}$, except that it makes the target address private before the assignment, as in:

$$
(\langle H\rangle ! \mathrm{w}):=\text { false }
$$

which allows this function to complete without information leaks. In particular, the revised assignment now updates y to $\mathrm{false}^{P}$, and so the return value is marked as partially leaked.

\section{TERMINATION-INSENSITIVE NON-INTERFERENCE}

We now verify that the permissive-upgrade strategy guarantees termination-insensitive non-interference.

Traditional non-interference arguments are based on an equivalence relation between labeled values that considers privately labeled values to be equivalent, even if the underlying raw values differ. The introduction of partially leaked data complicates this equivalence relation, since true ${ }^{L}$ and false ${ }^{P}$ are equivalent, as are false ${ }^{P}$ and $\mathrm{false}^{L}$, since in each case the label $P$ correctly identifies private data that is partially leaked. However, true ${ }^{L}$ and $\mathrm{false}^{L}$ are not equivalent, and so our desired "equivalence" relation does not satisfy transitivity.

Instead, we call this relation compatibility $(\sim)$. Intuitively, two stores are compatible if they differ only on private data, and executions that start with compatible stores should yield compatible results. In more detail, we define the compatibility relation $(\sim)$ on labels, values, substitutions, and stores as follows.

- Two labels are compatible if both are private or one is partially leaked:

$$
k_{1} \sim k_{2} \stackrel{\text { def }}{=}\left(k_{1}, k_{2}\right) \in\{(H, H),(P,-),(-, P)\}
$$

Label compatibility is neither reflexive (as $L \nsim L$ ) nor transitive (as $L \sim P \sim L$ but $L \not L$ ).

- Two values are compatible if either their labels are compatible or the labels are identical and the raw values are compatible.

$$
r_{1}^{k_{1}} \sim r_{2}^{k_{2}} \stackrel{\text { def }}{=} \quad k_{1} \sim k_{2} \vee\left(k_{1}=k_{2} \wedge r_{1} \sim r_{2}\right)
$$

- Two raw values are compatible if they are identical or they are both closures with identical code and compatible substitutions:

$$
\begin{aligned}
& r_{1} \sim r_{2} \stackrel{\text { def }}{=} \\
& r_{1}=r_{2} \vee\left(r_{1}=\left(\lambda x . e, \theta_{1}\right) \wedge r_{2}=\left(\lambda x . e, \theta_{2}\right) \wedge \theta_{1} \sim \theta_{2}\right)
\end{aligned}
$$

- Two substitutions are compatible (written $\theta_{1} \sim \theta_{2}$ ) if they have the same domain and compatible values:

$$
\begin{aligned}
\theta_{1} & \sim \theta_{2} \stackrel{\text { def }}{=} \\
& \operatorname{dom}\left(\theta_{1}\right)=\operatorname{dom}\left(\theta_{2}\right) \wedge \forall x \in \operatorname{dom}\left(\theta_{1}\right) .\left(\theta_{1}(x) \sim \theta_{2}(x)\right)
\end{aligned}
$$

- Two stores $\sigma_{1}$ and $\sigma_{2}$ are compatible (written $\sigma_{1} \sim \sigma_{2}$ ) if they are compatible at all common addresses:

$$
\sigma_{1} \sim \sigma_{2} \stackrel{\text { def }}{=} \forall a \in\left(\operatorname{dom}\left(\sigma_{1}\right) \cap \operatorname{dom}\left(\sigma_{2}\right)\right) . \sigma_{1}(a) \sim \sigma_{2}(a)
$$

We also introduce an evolution (or can evolve to) relation $(\sim)$ that constrains how evaluation with a private program counter can update the store. This relation composes in a transitive manner with compatibility: see Lemma 6 below.

- Label $k_{1}$ can evolve to $k_{2}$ if both labels are private or $k_{2}$ is partially leaked:

$$
k_{1} \leadsto k_{2} \stackrel{\text { def }}{=} \quad k_{1}=k_{2}=H \quad \vee \quad k_{2}=P
$$

- A value $r_{1}^{k_{1}}$ can evolve to $r_{2}^{k_{2}}$ if either the two values are equal or $k_{1}$ can evolve to $k_{2}$ :

$$
r_{1}^{k_{1}} \leadsto r_{2}^{k_{2}} \stackrel{\text { def }}{=} \quad r_{1}^{k_{1}}=r_{2}^{k_{2}} \quad \vee \quad k_{1} \leadsto k_{2}
$$

- A store $\sigma_{1}$ can evolve to $\sigma_{2}$ if every value in $\sigma_{1}$ can evolve to the corresponding value in $\sigma_{2}$ :

$$
\begin{aligned}
\sigma_{1} & \leadsto \sigma_{2} \stackrel{\text { def }}{=} \\
\operatorname{dom}\left(\sigma_{1}\right) & \subseteq \operatorname{dom}\left(\sigma_{2}\right) \wedge \forall a \in \operatorname{dom}\left(\sigma_{1}\right) . \sigma_{1}(a) \leadsto \sigma_{2}(a)
\end{aligned}
$$




\begin{tabular}{l|c|c}
\multirow{2}{*}{ Function $\mathrm{h}(\mathrm{x})$} & \multicolumn{2}{|c}{ Permissive Upgrade } \\
\cline { 2 - 3 } let $\mathrm{y}=$ ref true in & $\mathrm{x}=$ false $^{H}$ & $\mathrm{y}=$ true $^{H}$ \\
let $\mathrm{z}=$ ref true in & $\mathrm{z}=$ true $^{L}$ & $\mathrm{y}=$ true $^{L}$ \\
let $\mathrm{w}=$ ref $\mathrm{y}$ in & $\mathrm{w}=\mathrm{y}^{L}$ & $\mathrm{true}$ \\
if $(\mathrm{x})$ & branch not taken & $p c=H$ \\
$\quad$ then $\mathrm{w}:=\mathrm{z} ;$ & w remains $^{L}$ & w updated to $\mathrm{z}^{P}$ \\
(! $\mathrm{w}):=$ false; & $\mathrm{y}=$ false $^{L}$ & stuck \\
!y & returns false $^{L}$ & \\
Return Value: & false &
\end{tabular}

\begin{tabular}{|c|c|c|}
\hline \multirow[b]{2}{*}{ Function h_priv(x) } & \multicolumn{2}{|c|}{ Permissive Upgrade } \\
\hline & $\mathrm{x}=\mathrm{false} \mathrm{s}^{H}$ & $\mathrm{x}=\operatorname{true}^{H}$ \\
\hline let $y=$ ref true in & $\mathrm{y}=$ true $^{L}$ & $\mathrm{y}=$ true $^{L}$ \\
\hline let $z=$ ref true in & $\mathbf{z}=\operatorname{true}^{L}$ & $\mathbf{z}=\operatorname{true}^{L}$ \\
\hline let $\mathrm{w}=$ ref $\mathrm{y}$ in & $\mathrm{w}=y^{L}$ & $\mathrm{w}=y^{L}$ \\
\hline if $(x)$ & branch not taken & $p c=H$ \\
\hline then $\mathrm{w}:=\mathrm{z}$ & w remains $\mathrm{y}^{L}$ & w updated to $\mathbf{z}^{P}$ \\
\hline $\begin{array}{l}\langle\mathrm{H}\rangle(! \mathrm{w}):=\text { false; } \\
! \mathrm{y}\end{array}$ & $\begin{array}{l}\mathrm{y}=\mathrm{false} \mathrm{s}^{P} \\
\text { returns false } \mathrm{s}^{P}\end{array}$ & $\begin{array}{l}\mathbf{z}=\mathrm{false} \mathrm{e}^{P} \\
\text { returns true }\end{array}$ \\
\hline Return Value: & $\mathrm{false} \mathrm{e}^{P}$ & true $^{L}$ \\
\hline
\end{tabular}

The evolution relation captures how evaluation with a private program counter can update the store.

Lemma 1 (Evaluation Preserves Evolution). If $\sigma, \theta, e \Downarrow_{H} \sigma^{\prime}, v$ then $\sigma \leadsto \sigma^{\prime}$.

Proof. The proof proceeds by induction on the derivation of $\sigma, \theta, e \Downarrow_{H} \sigma^{\prime}, v$ and by case analysis on the final rule in the derivation.

- $[\mathrm{CONST}],[\mathrm{FUN}],[\mathrm{VAR}]: \sigma^{\prime}=\sigma$.

- $[\mathrm{APP}]$, [PRIM], [LABEL], [DEREF]: By induction.

- $[\mathrm{REF}]: \sigma$ and $\sigma^{\prime}$ agree on their common domain.

- [ASSign-PERmissive]: In this case, $e=\left(e_{1}:=e_{2}\right)$ and we have:

$$
\begin{gathered}
\sigma, \theta, e_{1} \Downarrow_{H} \sigma_{1}, a^{H} \\
\sigma_{1}, \theta, e_{2} \Downarrow_{H} \sigma_{2}, v \\
l=\operatorname{label}\left(\sigma_{2}(a)\right) \\
m=\operatorname{lift}(H, l) \\
\sigma^{\prime}=\sigma_{2}[a:=(v \sqcup m)]
\end{gathered}
$$

By induction, $\sigma \leadsto \sigma_{1} \leadsto \sigma_{2}$. By Lemma 2 below, $l \leadsto m$. Hence $\sigma_{2}(a) \leadsto(v \sqcup m)$ and so $\sigma_{2} \leadsto \sigma^{\prime}$.

In order to prove Lemma 1, we note some important properties of the $\sim$ relation. The evolution relation is transitive, and it is reflexive for both values and stores.

LEMma 2. $\forall m . m \leadsto \operatorname{lift}(H, m)$.

LEMMA 3. $\leadsto$ is transitive.

LEMMA 4. $\leadsto$ on values and stores is reflexive.

The evolution relation on values interacts in a "transitive" manner with the compatibility relation.

LEMMA 5. If $v_{1} \sim v_{2} \leadsto v_{3}$ then $v_{1} \sim v_{3}$.

If two stores are compatible $\left(\sigma_{1} \sim \sigma_{2}\right)$, then evolution of one store $\left(\sigma_{2} \leadsto \sigma_{3}\right)$ results in a new store that is compatible to the original stores $\left(\sigma_{1} \sim \sigma_{3}\right)$, with the caveat that any newly allocated address must not be in the original stores.
Lemma 6 (Evolution Preserves Compatibility of Stores). If $\sigma_{1} \sim \sigma_{2} \leadsto \sigma_{3}$ and $\left(\operatorname{dom}\left(\sigma_{1}\right) \backslash \operatorname{dom}\left(\sigma_{2}\right)\right) \cap \operatorname{dom}\left(\sigma_{3}\right)=\emptyset$ then $\sigma_{1} \sim \sigma_{3}$.

Proof. Let $D=\operatorname{dom}\left(\sigma_{1}\right) \cap \operatorname{dom}\left(\sigma_{3}\right)$. Then $D \sqsubseteq$ $\operatorname{dom}\left(\sigma_{2}\right)$. This means that $\forall a \in D . \sigma_{1}(a) \sim \sigma_{2}(a)$ and $\sigma_{2}(a) \leadsto \sigma_{3}(a)$. Therefore, by Lemma 5 :

$$
\forall a \in D . \sigma_{1}(a) \sim \sigma_{3}(a)
$$

Hence by the definition of the evolution relation, $\sigma_{1} \sim \sigma_{3}$.

Next, we first observe certain properties of labels. First, if two labels $k_{1}$ and $k_{2}$ are compatible, then joining any label to $k_{1}$ will still maintain the compatibility relation.

LEMma 7. If $k_{1} \sim k_{2}$ then $\left(k_{1} \sqcup l_{1}\right) \sim k_{2}$.

Also, if two labels are compatible and are part of different values, those values will also be compatible.

Lemma 8. If $k_{1} \sim k_{2}$ then $\left(v_{1} \sqcup k_{1}\right) \sim\left(v_{2} \sqcup k_{2}\right)$.

In a secure context ( $H$ as the first argument to the lift function), all labels are compatible.

Lemma 9. $\operatorname{lift}\left(H, l_{1}\right) \sim \operatorname{lift}\left(H, l_{2}\right)$.

Finally, we prove our central result: if an expression $e$ is executed twice from compatible stores and compatible substitutions, then both executions will yield compatible resulting stores and values. That is, private inputs never leak into public outputs.

Theorem 2 (Termination-Insensitive Non-Interference). Suppose $p c \in\{L, H\}$ and $\sigma_{1} \sim \sigma_{2}$ and $\theta_{1} \sim \theta_{2}$ and $\sigma_{i}, \theta_{i}, e \Downarrow_{p c}$ $\sigma_{i}^{\prime}, v_{i}$ for $i \in 1,2$. Then $\sigma_{1}^{\prime} \sim \sigma_{2}^{\prime}$ and $v_{1} \sim v_{2}$.

Proof. The proof is by induction on the derivation $\sigma_{1}, \theta_{1}, e \Downarrow_{p c} \sigma_{1}^{\prime}, v_{1}$ and case analysis on the last rule used in that derivation.

- [CONST]: Then $e=c$ and $\sigma_{1}^{\prime}=\sigma_{1} \sim \sigma_{2}=\sigma_{2}^{\prime}$ and $v_{1}=v_{2}=c^{p c}$.

- [VAR]: Then $e=x$ and $\sigma_{1}^{\prime}=\sigma_{1} \sim \sigma_{2}=\sigma_{2}^{\prime}$ and $v_{1}=$ $\left(\theta_{1}(x) \sqcup p c\right) \sim\left(\theta_{2}(x) \sqcup p c\right)=v_{2}$. 
- [FUn]: Then $e=\lambda x \cdot e^{\prime}$ and $\sigma_{1}^{\prime}=\sigma_{1} \sim \sigma_{2}=\sigma_{2}^{\prime}$ and $v_{1}=\left(\lambda x \cdot e^{\prime}, \theta_{1}\right)^{p c} \sim\left(\lambda x \cdot e^{\prime}, \theta_{2}\right)^{p c}=v_{2}$.

- [LABeL]: Then $e=\langle H\rangle e^{\prime}$. From the antecedent of this rule, we have that for $i \in 1,2$ :

$$
\sigma_{i}, \theta_{i}, e^{\prime} \Downarrow_{p c} \sigma_{i}^{\prime}, r_{i}^{k_{i}}
$$

By induction, $\sigma_{1}^{\prime} \sim \sigma_{2}^{\prime}$. Also, regardless of the raw values $r_{1}$ and $r_{2}, r_{1}^{H} \sim r_{2}^{H}$ by the definition of the compatibility relation.

- $[\mathrm{APP}]:$ In this case, $e=\left(e_{a} e_{b}\right)$, and from the antecedents of this rule, we have that for $i \in 1,2$ :

$$
\begin{gathered}
\sigma_{i}, \theta_{i}, e_{a} \Downarrow_{p c} \sigma_{i}^{\prime \prime},\left(\lambda x . e_{i}, \theta_{i}^{\prime}\right)^{k_{i}} \\
k_{i} \neq P \\
\sigma_{i}^{\prime \prime}, \theta_{i}, e_{b} \Downarrow_{p c} \sigma_{i}^{\prime \prime \prime}, v_{i}^{\prime} \\
\sigma_{i}^{\prime \prime \prime}, \theta_{i}^{\prime}\left[x:=v_{i}^{\prime}\right], e_{i} \Downarrow_{k_{i}} \sigma_{i}^{\prime}, v_{i}
\end{gathered}
$$

By induction:

$$
\begin{aligned}
\sigma_{1}^{\prime \prime} & \sim \sigma_{2}^{\prime \prime} \\
\sigma_{1}^{\prime \prime \prime} & \sim \sigma_{2}^{\prime \prime \prime} \\
\left(\lambda x . e_{1}, \theta_{1}^{\prime}\right)^{k_{1}} & \sim\left(\lambda x . e_{2}, \theta_{2}^{\prime}\right)^{k_{2}} \\
v_{1}^{\prime} & \sim v_{2}^{\prime}
\end{aligned}
$$

- If $k_{1}$ and $k_{2}$ are both $H$ then $v_{1} \sim v_{2}$, since they both have label at least $H$. By Lemma $1, \sigma_{i}^{\prime \prime \prime} \sim$ $\sigma_{i}^{\prime}$. Without loss of generality, we assume that the two executions allocate reference cells from disjoint parts of the address space, ${ }^{2}$ i.e.:

$$
\left(\operatorname{dom}\left(\sigma_{i}^{\prime}\right) \backslash \operatorname{dom}\left(\sigma_{i}^{\prime \prime \prime}\right)\right) \cap \operatorname{dom}\left(\sigma_{3-i}^{\prime}\right)=\emptyset
$$

Under this assumption, by Lemma $6 \sigma_{1}^{\prime \prime \prime} \sim \sigma_{2}^{\prime}$. Applying Lemma 6 again gives $\sigma_{1}^{\prime} \sim \sigma_{2}^{\prime}$.

- Otherwise $\theta_{1}^{\prime} \sim \theta_{2}^{\prime}$ and $e_{1}=e_{2}$ and $k_{1}=k_{2}$. By induction, $\sigma_{1}^{\prime} \sim \sigma_{2}^{\prime}$ and $v_{1}^{\prime \prime} \sim v_{2}^{\prime \prime}$, and hence $v_{1}^{\prime} \sim v_{2}^{\prime}$.

- [PRIM]: In this case, $e=\left(e_{a} e_{b}\right)$, and from the antecedents of this rule, we have that for $i \in 1,2$ :

$$
\begin{gathered}
\sigma_{i}, \theta_{i}, e_{a} \Downarrow_{p c} \sigma_{i}^{\prime \prime}, c_{i}^{k_{i}} \\
\sigma_{i}^{\prime \prime}, \theta_{i}, e_{a} \Downarrow_{p c} \sigma_{i}^{\prime}, d_{i}^{l_{i}} \\
r_{i}=\llbracket c_{i} \rrbracket\left(d_{i}\right)
\end{gathered}
$$

By induction:

$$
\begin{aligned}
\sigma_{1}^{\prime \prime} & \sim \sigma_{2}^{\prime \prime} & \sigma_{1}^{\prime} & \sim \sigma_{2}^{\prime} \\
c_{1}^{k_{1}} & \sim c_{2}^{k_{2}} & d_{1}^{l_{1}} & \sim d_{2}^{l_{2}}
\end{aligned}
$$

- If either $k_{1} \sim k_{2}$ or $l_{1} \sim l_{2}$, then by Lemma 7 $k_{1} \sqcup l_{1} \sim k_{2} \sqcup l_{2}$. Therefore, $r_{1}^{k_{1} \sqcup l_{1}} \sim r_{2}^{k_{2} \sqcup l_{2}}$.

- Otherwise, $r_{1}=r_{2}$, since $c_{1}=c_{2}$ and $d_{1}=d_{2}$. Also, $k_{1} \sqcup l_{1}=k_{2} \sqcup l_{2}$. Therefore, $r_{1}^{k_{1} \sqcup l_{1}} \sim r_{2}^{k_{2} \sqcup l_{2}}$.

- [REF]: In this case, $e=$ ref $e^{\prime}$. Without loss of generality, we assume that both evaluations allocate at the same address $a \notin \operatorname{dom}\left(\sigma_{1}\right) \cup \operatorname{dom}\left(\sigma_{2}\right)$, and so $a^{p c}=v_{1}=v_{2}$. From the antecedents of this rule, we have that for $i \in 1,2$ :

$$
\begin{gathered}
\sigma_{i}, \theta_{i}, e^{\prime} \Downarrow_{p c} \sigma_{i}^{\prime \prime}, v_{i}^{\prime} \\
\sigma_{i}^{\prime}=\sigma_{i}^{\prime \prime}\left[a:=v_{i}^{\prime}\right]
\end{gathered}
$$

By induction, $\sigma_{1}^{\prime \prime} \sim \sigma_{2}^{\prime \prime}$ and $v_{1}^{\prime} \sim v_{2}^{\prime}$, and so $\sigma_{1}^{\prime} \sim \sigma_{2}^{\prime}$.

\footnotetext{
${ }^{2}$ We refer the interested reader to [6] for an alternative proof argument that does use of this assumption, but which involves a more complicated compatibility relation on stores.
}

- [DEREF]: In this case, $e=! e^{\prime}$, and from the antecedents of this rule, we have that for $i \in 1,2$ :

$$
\begin{gathered}
\sigma_{i}, \theta_{i}, e^{\prime} \Downarrow_{p c} \sigma_{i}^{\prime}, a_{i}^{k_{i}} \\
v_{i}=\sigma_{i}^{\prime}\left(a_{i}\right) \sqcup k_{i}
\end{gathered}
$$

By induction, $\sigma_{1}^{\prime} \sim \sigma_{2}^{\prime}$ and $a_{1}^{k_{1}} \sim a_{2}^{k_{2}}$.

- Suppose $a_{1}^{k_{1}}=a_{2}^{k_{2}}$. Then $a_{1}=a_{2}$ and $k_{1}=k_{2}$ and $\sigma_{1}^{\prime}\left(a_{1}\right) \sim \sigma_{2}^{\prime}\left(a_{2}\right)$, and so $v_{1} \sim v_{2}$.

- Suppose $a_{1}^{k_{1}} \neq a_{2}^{k_{2}}$. Then since $a_{1}^{k_{1}} \sim a_{2}^{k_{2}}$ we must have that $k_{1} \sim k_{2}$ and hence $v_{1} \sim v_{2}$ from Lemma 8.

- [ASSign-PERmissive] In this case, $e=\left(e_{a}:=e_{b}\right)$, and from the antecedents of this rule, we have that for $i \in 1,2$ :

$$
\begin{gathered}
\sigma_{i}, \theta_{i}, e_{a} \Downarrow_{p c} \sigma_{i}^{\prime \prime}, a_{i}^{k_{i}} \\
\sigma_{i}^{\prime \prime}, \theta_{i}, e_{b} \Downarrow_{p c} \sigma_{i}^{\prime \prime \prime}, v_{i} \\
k_{i} \neq P \\
m_{i}=\operatorname{lift}\left(k_{i}, \operatorname{label}\left(\sigma_{i}^{\prime \prime \prime}\left(a_{i}\right)\right)\right) \\
\sigma_{i}^{\prime}=\sigma_{i}^{\prime \prime \prime}\left[a_{i}:=v_{i} \sqcup m_{i}\right]
\end{gathered}
$$

By induction:

$$
\begin{aligned}
\sigma_{1}^{\prime \prime} & \sim \sigma_{2}^{\prime \prime} & \sigma_{1}^{\prime \prime \prime} & \sim \sigma_{2}^{\prime \prime \prime} \\
a_{1}^{k_{1}} & \sim a_{2}^{k_{2}} & v_{1} & \sim v_{2}
\end{aligned}
$$

- If $k_{1} \sim k_{2}$ then $k_{1}=k_{2}=H$. By Lemma 9, $m_{1} \sim m_{2}$. By Lemma 8, $\left(v_{1} \sqcup m_{1}\right) \sim\left(v_{2} \sqcup m_{2}\right)$. Hence $\sigma_{1}^{\prime} \sim \sigma_{2}^{\prime}$.

- Otherwise $k_{1}=k_{2}=L$. Then $m_{1}=m_{2}=L$ and hence $\sigma_{1}^{\prime} \sim \sigma_{2}^{\prime}$.

\section{PRIVATIZATION INFERENCE}

The permissive-upgrade semantics guarantees terminationinsensitive non-interference while getting stuck on fewer programs than the NSU semantics, and it will not get stuck if the program includes privatization operations on sensitive uses of partially leaked data.

We now extend our semantics to infer these privatization operations. We begin by adding a position marker $p \in$ Position on each sensitive operation (applications and assignments) where partially leaked data is not permitted.

$$
e::=\ldots\left|\left(e_{1} e_{2}\right)^{p}\right|\left(e_{1}:=e_{2}\right)^{p}
$$

Rather than explicitly insert privatization operations at particular positions in the source code, we instead extend the store $\sigma$ to now also record the positions where these operations have been conceptually inserted.

We replace the original [APP] evaluation rule with three variants, and similarly for [ASSIGN-PERMISSIVE], as shown in Figure 8 . The [APP-NORMAL] rule applies if a privatization operation has not been inserted $(p \notin \sigma)$ and is not needed $(k \neq P)$. [APP-UPGRADE] handles situations where the privatization operation has been inserted $(p \in \sigma)$ by ignoring the label $k$ on the closure and behaving as if the closure were labeled private instead. [APP-INFER] handles situations where a privatization operation is required $(k=P)$ but has not yet been inserted $(p \notin \sigma)$; it adds this position tag to the store (conceptually inserting the required privatization operation) and then reevaluates the application. 


\section{Evaluation Rules: $\quad \sigma, \theta, e \Downarrow_{p c} \sigma^{\prime}, v$}

[APP-NORMAL]

$$
\begin{gathered}
p \notin \sigma \\
\sigma, \theta, e_{1} \Downarrow_{p c} \sigma_{1},\left(\lambda x . e, \theta^{\prime}\right)^{k} \\
k \neq P \\
\sigma_{1}, \theta, e_{2} \Downarrow_{p c} \sigma_{2}, v_{2} \\
\frac{\sigma_{2}, \theta^{\prime}\left[x:=v_{2}\right], e \Downarrow_{k} \sigma^{\prime}, v}{\sigma, \theta,\left(e_{1} e_{2}\right)^{p} \Downarrow_{p c} \sigma^{\prime}, v}
\end{gathered}
$$

[APP-UPGRADE]

$$
\begin{gathered}
p \in \sigma \\
\sigma, \theta, e_{1} \Downarrow_{p c} \sigma_{1},\left(\lambda x . e, \theta^{\prime}\right)^{k} \\
\sigma_{1}, \theta, e_{2} \Downarrow_{p c} \sigma_{2}, v_{2} \\
\frac{\sigma_{2}, \theta^{\prime}\left[x:=v_{2}\right], e \Downarrow_{H} \sigma^{\prime}, v}{\sigma, \theta,\left(e_{1} e_{2}\right)^{p} \Downarrow_{p c} \sigma^{\prime}, v}
\end{gathered}
$$

[APP-INFER]

$$
\begin{gathered}
p \notin \sigma \\
\sigma, \theta, e_{1} \Downarrow_{p c} \sigma_{1},\left(\lambda x . e, \theta^{\prime}\right)^{k} \\
k=P \\
\frac{(\sigma \cup\{p\}), \theta,\left(e_{1} e_{2}\right)^{p} \Downarrow_{p c} \sigma^{\prime}, v}{\sigma, \theta,\left(e_{1} e_{2}\right)^{p} \Downarrow_{p c} \sigma^{\prime}, v}
\end{gathered}
$$

$$
\begin{gathered}
\text { [ASSIGN-NORMAL] } \\
p \notin \sigma \\
\sigma, \theta, e_{1} \Downarrow_{p c} \sigma_{1}, a^{k} \\
k \neq P \\
\sigma_{1}, \theta, e_{2} \Downarrow_{p c} \sigma_{2}, v \\
l=\operatorname{lift}\left(k, \text { label }\left(\sigma_{2}(a)\right)\right) \\
\frac{l, \theta,\left(e_{1}:=e_{2}\right)^{p} \Downarrow_{p c} \sigma_{2}[a:=(v \sqcup l)], v}{\sigma}
\end{gathered}
$$

[ASSIGN-UPGRADE]

$$
\begin{gathered}
p \in \sigma \\
\sigma, \theta, e_{1} \Downarrow_{p c} \sigma_{1}, a^{k} \\
\sigma_{1}, \theta, e_{2} \Downarrow_{p c} \sigma_{2}, v \\
l=\operatorname{lift}\left(H, \operatorname{label}\left(\sigma_{2}(a)\right)\right) \\
\frac{l, \theta,\left(e_{1}:=e_{2}\right)^{p} \Downarrow_{p c} \sigma_{2}[a:=(v \sqcup l)], v}{}
\end{gathered}
$$

[ASSIGN-INFER]

$$
\begin{gathered}
p \notin \sigma \\
\sigma, \theta, e_{1} \Downarrow_{p c} \sigma_{1}, a^{k} \\
k=P \\
\frac{(\sigma \cup\{p\}), \theta,\left(e_{1}:=e_{2}\right)^{p} \Downarrow_{p c} \sigma^{\prime}, v}{\sigma, \theta,\left(e_{1}:=e_{2}\right)^{p} \Downarrow_{p c} \sigma^{\prime}, v}
\end{gathered}
$$

Our revised semantics still guarantees non-interference, but only if the evaluation did not infer additional privatization operations. This observation leads to some interesting design decisions. If output of the final result is allowed even when there was an inferred label, then non-interference is not guaranteed, but the information leak is detected. If output is forbidden in this case, then the behavior is identical to the permissive-upgrade semantics.

Theorem 3 (Non-Interference of Privatization Inference). Suppose $p c \neq P$ and $\sigma_{1} \sim \sigma_{2}$ and $\theta_{1} \sim \theta_{2}$ and $\sigma_{i}, \theta_{i}, e \Downarrow_{p c}$ $\sigma_{i}^{\prime}, v_{i}$ and $P_{i}=\left(\sigma_{i}^{\prime} \backslash \sigma_{i}\right) \cap$ Position for $i \in 1,2$. If $P_{1}=P_{2}=\emptyset$ then $\sigma_{1}^{\prime} \sim \sigma_{2}^{\prime}$ and $v_{1} \sim v_{2}$.

We next show that adding some labels $A$ to a program only influences the labels in the program's result, but not the raw values. To formalize this property, we introduce a raw equivalence order $(\approx)$ that identifies values, substitutions, and stores that differ only in their labels, not in their underlying raw values. Moreover, raw equivalent stores are allowed to differ in the position tags that they include, i.e., $\sigma \approx(\sigma \cup A)$.

Theorem 4 (Non-Interference Of Privatization Operations). Suppose $p c \neq P$ and $A \subseteq$ Position and $\sigma, \theta, e \Downarrow_{p c} \sigma_{1}, v_{1}$ and $(\sigma \cup A), \theta, e \Downarrow_{p c} \sigma_{2}, v_{2}$. Then $\sigma_{1} \approx \sigma_{2}$ and $v_{1} \approx v_{2}$

We prove this theorem via the following lemma, which strengthens the inductive hypothesis.

Lemma 10. Suppose $p c \neq P$ and $\sigma_{1} \approx \sigma_{2}$ and $\theta_{1} \approx \theta_{2}$ and $\sigma_{i}, \theta_{i}, e \Downarrow_{p c_{i}} \sigma_{i}^{\prime}, v_{i}$ for $i \in 1,2$. Then $\sigma_{1}^{\prime} \approx \sigma_{2}^{\prime}$ and $v_{1} \approx v_{2}$.
Proofs for Theorem 3 and Lemma 10 are available in a related technical report [5].

Whenever a program occurs that surrenders a bit of information, the missing privatization operation can be determined. These inferred operations might lead to label creep, especially if a function is used both with public data and private data. Addressing this issue remains future work.

\section{RELATED WORK}

Denning's papers $[12,13]$ are largely the beginning of information flow analysis for a high-level language. Her work advocates a static certification approach; since then, static approaches have dominated because of their generally superior performance and the perceived advantages in handling implicit flows. Volpano et al. [41] and Heintze and Riecke [21] are two of the most well known type-based approaches, though their target languages are relatively minimal. Pottier and Simonet [31] introduce a more complex system for Core ML. Chaudhuri et al. [8] create a type system for handling explicit flows in Windows Vista. Sabelfeld and Myers [35] give an excellent overview of different languagebased information flow analyses.

Recently, there has been more appreciation of the complementary benefits that each approach offers. Many strategies rely primarily on static techniques and insert dynamic runtime checks only in ambiguous cases [7,39]. This approach reduces false positives with a minimum impact on performance. Myers [30] introduced JFlow, a variant of Java using this hybrid strategy, which was the basis for Jif [23]. Chugh et al. [11] propose a mostly static approach for analyzing 
JavaScript with "holes" for dynamically generated code.

Generally, dynamic analysis is more often applied to clientside scripting, particularly for JavaScript, where dynamic typing makes type-based approaches difficult. Vogt et al. [40] reverse the standard hybrid approach, relying primarily on dynamic checks but falling back to runtime certification for implicit flows. Chudnov and Naumann [10] inline a hybrid information flow monitor, again with a focus on JavaScript.

Several papers address challenges that are of particular interest to JavaScript. Russo et al. study information flow analysis in the DOM [34] and timeout mechanisms [32]. Askarov and Sabelfeld [3] cover declassification and analysis of dynamic code evaluation. Magazinius et al. [26] study safe declassification in JavaScript mashups.

In his dissertation, Zdancewic [42] first proposed rules for dynamic analysis to effectively handle implicit flows. Our own work later dubbed the key assignment rule the nosensitive-upgrade check and addressed performance concerns for dynamic analysis with a sparse-labeling approach [4]. Le Guernic et al. [19] use dynamic automaton-based monitoring. Sabelfeld and Russo [36] formally prove that both static and dynamic approaches make the same security guarantees. Shroff et al. [38] dynamically track dependencies to guarantee noninterference. Shinnar et al. [37] provide a dynamic analysis that follows a lazy policy enforcement, similar in spirit to our permissive-upgrades. This same paper also discusses the interplay between different dimensions of information, focusing primarily on integrity and confidentiality.

Flow-sensitive information flow analyses attempt to reduce false-positives. Hunt and Sands [22] use a flow-sensitive type-system while Hammer and Snelting [20] use program dependency graphs. Russo and Sabelfeld discuss the limits of flow-sensitivity for purely dynamic languages [33].

Several papers highlight important areas for concern for any production-worthy information flow analysis. Both Chong and Myers [9] and Fournet and Rezk [16] focus on downgrading confidential information. Askarov et al. [1] demonstrate that Denning-style analysis may leak more than one bit in the presence of intermediary output channels, but that any attack will be limited to a brute-force approach. Askarov and Sabelfeld [2] and King et al. [24] discuss exception handling challenges. Livshits et al. [25] design a system for inferring information flow policies to handle explicit flows.

\section{CONCLUSION}

We present a permissive-upgrade semantics that tracks information flow in a more flexible manner than prior dynamic approaches, using a new label $(P)$ to permit partially leaked data without loss of soundness. Using this strategy, we introduce a degree of flow-sensitivity into dynamic information flow analysis. To avoid stuck executions, privatization operations are required on sensitive uses of partially leaked data, and we show how these labels can be inferred dynamically. We hope these techniques will help enforce important information-flow policies in dynamically typed web applications. We are exploring how to incorporate these and other ideas into the Firefox web browser [14].

\section{References}

[1] A. Askarov, S. Hunt, A. Sabelfeld, and D. Sands. Termination-insensitive noninterference leaks more than just a bit. In ESORICS '08: Proceedings of the 13th European Symposium on Research in Computer Security, pages 333-348, Berlin, Heidelberg, 2008. Springer-Verlag.

[2] A. Askarov and A. Sabelfeld. Catch me if you can: permissive yet secure error handling. In PLAS '09: Proceedings of the ACM SIGPLAN Fourth Workshop on Programming Languages and Analysis for Security, pages 45-57, New York, NY, USA, 2009. ACM.

[3] A. Askarov and A. Sabelfeld. Tight enforcement of information-release policies for dynamic languages. In IEEE Computer Security Foundations Symposium, pages 43-59, Washington, DC, USA, 2009. IEEE Computer Society.

[4] T. H. Austin and C. Flanagan. Efficient purely-dynamic information flow analysis. In PLAS '09: Proceedings of the ACM SIGPLAN Fourth Workshop on Programming Languages and Analysis for Security, pages 113-124, New York, NY, USA, 2009. ACM.

[5] T. H. Austin and C. Flanagan. Permissive dynamic information flow analysis. Technical Report UCSC-SOE09-34, The University of California at Santa Cruz, 2009.

[6] A. Banerjee and D. A. Naumann. Secure information flow and pointer confinement in a java-like language. In IEEE Computer Security Foundations Workshop, pages 253-267. IEEE Computer Society, 2002.

[7] D. Chandra and M. Franz. Fine-grained information flow analysis and enforcement in a java virtual machine. In $A C S A C$, pages 463-475. IEEE Computer Society, 2007.

[8] A. Chaudhuri, P. Naldurg, and S. K. Rajamani. A type system for data-flow integrity on windows vista. In Ú. Erlingsson and M. Pistoia, editors, PLAS, pages 89-100. ACM, 2008.

[9] S. Chong and A. C. Myers. Security policies for downgrading. In $C C S$ '04: Proceedings of the 11th $A C M$ conference on Computer and communications security, pages 198-209, New York, NY, USA, 2004. ACM.

[10] A. Chudnov and D. A. Naumann. Information flow monitor inlining. In IEEE Computer Security Foundations Symposium. IEEE Computer Society, 2010.

[11] R. Chugh, J. A. Meister, R. Jhala, and S. Lerner. Staged information flow for javascript. In $P L D I$ '09: Proceedings of the 2009 ACM SIGPLAN conference on Programming language design and implementation, pages 50-62, New York, NY, USA, 2009. ACM.

[12] D. E. Denning. A lattice model of secure information flow. Communications of the ACM, 19(5):236-243, 1976.

[13] D. E. Denning and P. J. Denning. Certification of programs for secure information flow. Communications of the ACM, 20(7):504-513, 1977.

[14] B. Eich. Mozilla FlowSafe: Information flow security for the browser. https://wiki.mozilla.org/FlowSafe, accessed October 2009.

[15] Developer's wiki: FBJS. http://wiki.developers. facebook.com/index.php/FBJS, accessed January 2010 .

[16] C. Fournet and T. Rezk. Cryptographically sound implementations for typed information-flow security. In Symposium on Principles of Programming Languages, pages 323-335, 2008. 
[17] A. Gal, B. Eich, M. Shaver, D. Anderson, B. Kaplan, G. Hoare, D. Mandelin, B. Zbarsky, J. Orendorff, M. Bebenita, M. Chang, M. Franz, E. Smith, R. Reitmaier, and M. Haghighat. Trace-based just-in-time type specialization for dynamic languages. In Conference on Programming Language Design and Implementation, 2009.

[18] Caja. http://code.google.com/p/google-caja/, accessed December 2009

[19] G. L. Guernic, A. Banerjee, T. P. Jensen, and D. A. Schmidt. Automata-based confidentiality monitoring. In M. Okada and I. Satoh, editors, ASIAN, volume 4435 of Lecture Notes in Computer Science, pages 7589. Springer, 2006.

[20] C. Hammer and G. Snelting. Flow-sensitive, contextsensitive, and object-sensitive information flow control based on program dependence graphs. International Journal of Information Security, 2009.

[21] N. Heintze and J. G. Riecke. The slam calculus: Programming with secrecy and integrity. In Symposium on Principles of Programming Languages, pages 365-377, 1998.

[22] S. Hunt and D. Sands. On flow-sensitive security types. In J. G. Morrisett and S. L. P. Jones, editors, POPL, pages 79-90. ACM, 2006.

[23] Jif homepage. http://www.cs.cornell.edu/jif/, accessed October 2009.

[24] D. King, B. Hicks, M. Hicks, and T. Jaeger. Implicit flows: Can't live with 'em, can't live without 'em. In International Conference on Information Systems Security, pages 56-70, 2008.

[25] V. B. Livshits, A. V. Nori, S. K. Rajamani, and A. Banerjee. Merlin: specification inference for explicit information flow problems. In M. Hind and A. Diwan, editors, PLDI, pages 75-86. ACM, 2009.

[26] J. Magazinius, A. Askarov, and A. Sabelfeld. A latticebased approach to mashup security. In Proceedings of the ACM Symposium on Information Computer and Communications Security, 2010.

[27] Internet explorer security zones. http://technet. microsoft.com/en-us/library/dd361896.aspx, accessed December 2009.

[28] JavaScript security in Mozilla. http://www.mozilla. org/projects/security/components/jssec.html, accessed January 2009.

[29] Same origin policy for JavaScript. https: //developer.mozilla.org/En/Same_origin_policy_ for_JavaScript, accessed January 2010.

[30] A. C. Myers. Jflow: Practical mostly-static information flow control. In Symposium on Principles of Programming Languages, pages 228-241, 1999.

[31] F. Pottier and V. Simonet. Information flow inference for ML. Transactions on Programming Languages and Systems, 25(1):117-158, 2003.

[32] A. Russo and A. Sabelfeld. Securing timeout instructions in web applications. In IEEE Computer Security Foundations Symposium, 2009.

[33] A. Russo and A. Sabelfeld. Dynamic vs. static flowsensitive security analysis. In IEEE Computer Security Foundations Symposium. IEEE Computer Society, 2010 .
[34] A. Russo, A. Sabelfeld, and A. Chudnov. Tracking information flow in dynamic tree structures. In M. Backes and P. Ning, editors, ESORICS, volume 5789 of Lecture Notes in Computer Science, pages 86-103. Springer, 2009.

[35] A. Sabelfeld and A. C. Myers. Language-based information-flow security. Selected Areas in Communications, IEEE Journal on, 21(1):5-19, Jan 2003.

[36] A. Sabelfeld and A. Russo. From dynamic to static and back: Riding the roller coaster of information-flow control research. In Perspectives of System Informatics, 2009 .

[37] A. Shinnar, M. Pistoia, and A. Banerjee. A language for information flow: dynamic tracking in multiple interdependent dimensions. In S. Chong and D. A. Naumann, editors, PLAS, pages 125-131. ACM, 2009.

[38] P. Shroff, S. F. Smith, and M. Thober. Dynamic dependency monitoring to secure information flow. In $C S F$, pages 203-217. IEEE Computer Society, 2007.

[39] V. N. Venkatakrishnan, W. Xu, D. C. DuVarney, and R. Sekar. Provably correct runtime enforcement of noninterference properties. In Information and Communications Security, pages 332-351, 2006.

[40] P. Vogt, F. Nentwich, N. Jovanovic, E. Kirda, C. Krügel, and G. Vigna. Cross site scripting prevention with dynamic data tainting and static analysis. In NDSS. The Internet Society, 2007.

[41] D. Volpano, C. Irvine, and G. Smith. A sound type system for secure flow analysis. Journal of Computer Security, 4(2-3):167-187, 1996.

[42] S. A. Zdancewic. Programming languages for information security. PhD thesis, Cornell University, Ithaca, NY, USA, 2002. Chair-Myers,, Andrew. 